\title{
Design and Simulation of a Very Fast and Compact All-Optical Full-Subtractor Based an Nonlinear Effect in 2D Photonic Crystals
}

\section{Reza Beiranvand}

Lorestan University

Ali Mir ( $\nabla$ mir.a@lu.ac.ir)

Lorestan University https://orcid.org/0000-0001-7196-6513

Reza Talebzadeh

Lorestan University

\section{Research Article}

Keywords: All-optical gates, full-Subtractor, nonlinear effects

Posted Date: May 10th, 2021

DOI: https://doi.org/10.21203/rs.3.rs-320958/v1

License: (c) (i) This work is licensed under a Creative Commons Attribution 4.0 International License.

Read Full License 


\section{Abstract}

In this paper, by using the non-linear effects and also destructive and constructive interferences between waveguides, we have designed and simulated an all-optical full-Subtractor based on two-dimensional photonic crystals. The proposed Subtractor has a very simple structure which is composed of $33 \times 31$ silicon rods immersed in air in a square lattice and involves three input ports (bits) and an additional waveguide to exhaust the unwanted light. We imposed some defect rods to control the behavior of the light. The used non-linear material, is a doped glass with $1.4 \times 10^{-14} \mathrm{~m}^{2} / \mathrm{w}$ non-linear refractive index which is very greater than the non-linearity refractive index of silicon, $3.46 \times 10^{-20} \mathrm{~m}^{2} / \mathrm{w}$. Since the proposed structure is very simple and compact, it can be applicable in optical integrated circuits and optical calculations.

\section{Introduction}

Photonic crystals (PCs) are a good candidate to design and fabricate the optical-gates in the range of the light wave wavelengths. PCs are periodic structures of dielectric materials which do not allow propagation of light in a range of wavelengths [1]. This feature of PCs is known as photonic bandgap (PBG) in which light in any angle and polarization is not allowed to propagate into them [2]. As a result, in the photonic crystals optical waveguides, light can propagate without scattering and attenuating. Many all-optical gates have been proposed based on this phenomenon such as optical filters [3-4], optical demultiplexers [5-10] and optical gates [11].

The combination of PCs with non-linear materials gave the opportunity to design all-optical gates with the desired features. Among these features is the possibility of integrating the all-optical systems on an all-optical chips. These chips can work in higher frequencies with lower consumption power compared to the nowadays silicon chips. Using the mentioned chips together with the optical interconnects would give the possibility of designing the modern all-optical computers with a much higher speed in digital calculations [12-14].

One of the key blocks for calculation is subtraction operation. Up to our knowledge, any all-optical fullSubtractor have not been designed based on photonic crystals. However, many attempts were done on the half-subtraction [15-18].

Moradi et al, proposed an all-optical half-Subtractor based on 2-D PCs [15]. Their proposed device consisted of $64 \times 31$ silicon rods in a hexagonal lattice with a lattice constant of $607 \mathrm{~nm}$. They used doped glass as a non-linear material with a refractive index of $1.4 \times 10^{-14} \mathrm{~m}^{2} / \mathrm{w}$ [15]. Askarian et al, proposed an all-optical half-Subtractor based on 2-D photonic crystals. They used beam interference to fulfill the behavior of their proposed system and controlled the light propagation by imposing two extra waveguides with different input powers [16]. Namdari et al, proposed an all-optical half-Subtractor based on linear materials. They just used $20 \times 20$ silicon rods in a hexagonal lattice. In order to control the light, they imposed four defect rods in their structure [17]. Parandin et al proposed a half-Subtractor using 
photonic crystals with a lattice constant of $a=640 \mathrm{~nm}$. Their basic structure consists of $19 \times 19$ dielectric rods in the air. They used different input powers for their input bits in a way that value for " $y$ " bit laser is 1.5 times higher than the " $x "$ bit and their input powers differ 20 degrees in their phases [18]. All of the mentioned literature reveals the need of designing an optical full-Subtractor. In this paper, we proposed an all-optical full-Subtractor based on two-dimensional photonic crystals. As far as we know, this is the first time that an all-optical Subtractor to be offered. We controlled the behavior of light by imposing just defect rods and one extra waveguide. We also utilized a ring resonator which can resonance based on the power it meets. The proposed gate is very simple and ultra-compact. The rest of the paper is organized as follows: In Sect. 2, the photonic bandgap will be calculated and then the full-Subtractor structure will be introduced. In Sect. 3, the results will be investigated and compared with those of previous literature; finally, the conclusion and simulation results will be discussed in Sect. 4 .

\section{The Proposed All-optical Full-subtractor 2. a) non-linear effects}

An appropriate way to define the nonlinear effect or intensity-dependent refractive index is by means of the equation [19].

$$
n(I)=n_{0}+n_{2} I
$$

where $n_{0}$ and $n_{2}$ are the linear and non-linear refractive indices of the non-linear material respectively and $I$ denotes the time-averaged intensity of the optical field. This feature is among the important properties of non-linear materials which can be applicable in designing optical systems. By increasing the magnitude of the input power, the refractive index of materials varies because of the third order polarization susceptibility by [19]:

$$
n_{2}=\frac{12 \pi^{2}}{n_{0}^{2} c} \chi^{(3)}
$$

where $\chi^{(3)}$ is the susceptibility, and $c$ is the speed of light in the vacuum. This phenomenon is known as "Kerr effect" and occurs in high input fields [20] for materials with third order susceptibility, $\chi^{(3)}$, and is a nonlinear effect. We used of this occurrence in our proposed device.

\section{2. b) PBG of the Subtractor}

We used of plane wave expansion method to calculate the PBG of the basic structure [21-22]. After that we calculate the desired frequencies by solving the Maxwell equations in the frequency domain. The basic structure that is used to design involved of $33 \times 31$ silicon dielectric rods immersed in the air. The mentioned number of rods is due to the sufficient space to create the input and output paths of the 
structure according to the number of bits used in an all-subtractor. The radii of the rods and the lattice constant were $116 \mathrm{~nm}$ and $580 \mathrm{~nm}$ respectively. The effective refractive index of silicon is 3.46 at the central wavelength of $1550 \mathrm{~nm}$ [23]. We selected the lattice constant in a way that the PBG locates in the third communication window. The schematic diagram of the photonic band gap is shown in Fig. 1 .

The band structure is calculated for TE polarization. As one can see in Fig. 1, there are two PBGs in the structure: $0.61<a / \lambda<0.66$ and $0.31<a / \lambda<0.44$. We selected the lower bandgap for our designing and based on the lattice constant of the proposed device, this bandgap is equal to $1310 \mathrm{~nm}<\lambda<1870 \mathrm{~nm}$ which is located in the third communication window. Therefore, the wavelength of the applied light source must be chosen in the PBG range so that they cannot enter the structure unless through a path is created for them using linear defects. Therefore, the wavelength of $1550 \mathrm{~nm}$ in the desired range is suitable for this structure.

\section{2. c) The proposed full-Subtractor}

Analogous to the digital full-Subtractor, the optical full-Subtractor has three input ports we named them "A", and "B" for the input bits and " $\mathrm{B}_{\text {in }}$ " for the input borrowed bit. It also has two output ports we named them " $D$ " for the difference and " $B_{\text {out }}$ " for the output borrow bit. The truth table of a full-Subtractor is shown in Table 1. We do the subtraction operation as "A- B".

Table 1. The truth table of a full-Subtractor.

\begin{tabular}{|lllll|}
\hline \multicolumn{3}{|c|}{ Input Bits } & \multicolumn{2}{c|}{ Output Bits } \\
\hline $\mathrm{A}$ & $\mathrm{B}$ & $\mathrm{B}_{\text {in }}$ & $\mathrm{D}$ & $\mathrm{B}_{\text {out }}$ \\
\hline 0 & 0 & 0 & 0 & 0 \\
0 & 0 & 1 & 1 & 1 \\
\hline 0 & 1 & 0 & 1 & 1 \\
\hline 0 & 1 & 1 & 0 & 1 \\
\hline 1 & 0 & 0 & 1 & 0 \\
\hline 1 & 0 & 1 & 0 & 0 \\
1 & 1 & 0 & 0 & 0 \\
\hline 1 & 1 & 1 & 1 & 1 \\
\hline
\end{tabular}

To design the Subtractor, first we created the input and output waveguides. The proposed all optical full Subtractor is consisted of the input ( $A, B$ and $\left.B_{\text {in }}\right)$ and output ( $D$ and $B_{\text {out }}$ ) waveguides, and the interface between them created inside the base structure of the PCs. As it can be seen from Fig. 2, the structure has 
three input bits and two output bits. The paths of lights are created by removing some of rods, proportional to the desired function. An extra waveguide, W2, is created below the "A" input bit to exhaust the idler signals and also to establish the symmetry in the inputs and the whole of the structure. It also synchronize the inputs for better interferences. A ring resonator, called R1, composed of non-linear materials (doped glass [26]), is created between the two W5 and W6 waveguides to construction coupling condition between them. The radii of the non-linear doped glass rods that are highlighted by blue color in Fig. 2, are $58 \mathrm{~nm}$, which we reached this amount after several times of trial and error as well as optimization. The ring resonator can resonate when the input power increases the threshold power that is needed for its excitation. The input power for all inputs is equal to $10 \mathrm{~mW} / \mu \mathrm{m}^{2}$.

When the input port of " $\mathrm{A}$ " is off, the behavior of incoming light from "B" and " $\mathrm{B}_{\text {in }}$ " ports are controlled by imposing additional R2 defect rods that can act as a filter for the input signals. The radii of these defects are $52 \mathrm{~nm}$ which is determined by numerical analysis and considering the optimal conditions for the normalized output powers by scanning different values. In the proposed structure, we considered the nonlinear properties of silicon too.

\section{Results And Discussion}

\section{3. a) Electric field distribution and spectrum response}

The most commonly used method of calculating the propagation of light in a structure is the finite difference time domain (FDTD) method [24]. We employed of $1550 \mathrm{~nm}$ Gaussian waves as inputs of RSoft software tool to study the propagation of light in eight different cases for our proposed structure based on the truth table of Subtractor (Table 1).

Case 1: when there is no input light, we have no power in the structure, so the outputs are zero. It is equivalent to the first row of Table 1.

Case 2: when "A"="B"=0 and " $B$ in $=1$, is equivalent to the second row of the truth table, the input light from $B_{\text {in }}$ propagates in W4 and W6 waveguides. When the input light reaches to the ring resonator (R1), it cannot provide the power needed for resonance and hence propagates toward the output ports. The normalized power at "D" and "B $\mathrm{But}_{\text {" }}$ ports are $83 \%$ and $65 \%$ respectively and can be considered logically "1". The electric field distribution (EFD) and output spectrum of this case are shown in Figs. 3. (a) and (b) respectively. However, we can see an amount of back propagation light in the input ports as loss.

Case 3: when "A" = "Bin" = 0 and "B"=1, equivalent to the third row of the truth table, and is similar to case 2. The input light from "B" port cannot excite the ring resonator, so propagates toward the output ports. The normalized power at the output ports of " $\mathrm{D}$ " and " $\mathrm{B}_{\text {out }}$ " are $83 \%$ and $65 \%$ respectively and can be considered as "1" logic. The EFD and output spectrum of this case are shown in Figs. 4 (a) and (b) respectively. 
Case 4: when "A"= 0 and "B" = "Bin $=$ "1", is equivalent to the $4^{\text {th }}$ row of Table 1 . Here, the optical signals from the two input ports propagate in the $\mathrm{W} 3$ and $\mathrm{W} 4$ waveguides and reach to the ring R1 resonator. Now, the optical intensity is sufficient enough to excite the R1 resonator. It couples the optical signal, both to the output ports and to the W5 and exhaust it from W1 and W2 waveguides. Simultaneously, since the R1 does not fully couple the light and a portion of light propagates to the output ports, the lights interfere destructively and cause no light to propagate to the "D" port. The role of defects, which are named R2, is seen in this case. They are responsible of creation a phase difference and hence constructive interference. Also they act as a filter. Consequently, the output normalized power in " $D$ " and "B out output ports are $5 \%$ and $92 \%$ respectively which can considered as " 0 " and " 1 " logically. The EFD and the output spectra in this case are shown in Figs. 5 (a) and (b) respectively.

Case 5: "A" =1 and "B" = "B $\mathrm{B}_{\text {in }}=0$. This case is equivalent to the $5^{\text {th }}$ row of the Subtractor truth table which the input light propagates through W1 and reaches to W5. Because of its insufficient intensity, cannot excite R1 ring resonator. So $73 \%$ of its power is directed to "D" output port and the rest is exhausted from W3 and W4. R2 Defect rods play their filtering role here, too. These rods do not allow the light to propagate in the " $\mathrm{B}_{\text {out }}$ " port. It is necessary to mention that the size and the location of these defect rods have been selected after many attempts in a try and error method. As a result, the normalized power in " $D$ " and " $\mathrm{B}_{\text {out }}$ " ports are $73 \%$ and $5 \%$ which can be considered as logically " 1 " and "0" respectively. The EFD and the spectrum response of this case are shown in Figs. 6 (a) and (b) respectively and verify the simulation.

Case 6: when "A" $=1, " B "=0$, and $" B_{\text {in }} "=1$, is equivalent to the $6^{\text {th }}$ row of the Subtractor truth table. In this case, because there are two inputs, the power of media near R1 ring resonator is sufficient enough to resonate. As a result, ring fully couples the light to W3 and it exits from the structure. The normalized power in the "D" and "B out are $6 \%$ and $2 \%$ respectively and can be considered logically "0". The EFD and the spectrum response of this case are shown in Figs. 7 (a) and (b) respectively.

Case 7: if "A"= "B" = "1" and " $\mathrm{B}_{\text {in }} "=0$, is equivalent to the $7^{\text {th }}$ row of the truth table and is similar to case 6 . Again we have two inputs and the power is high enough to resonance in the resonator. The ring couples the input optical power to W3 and it exits from the system. As a result, there would be no sufficient power at "D" and "B $\mathrm{B}_{\text {out }}$ " output ports. They are $6 \%$ and $2 \%$ respectively which can be considered logically " 0 ". The EFD and spectrum response in this case are shown in the Figs. 8 (a) and (b) respectively.

Case 8: when "A"="B"="B out"="1", we are in the last row of the truth table. In this case, the electric filed around the ring resonator is high enough. So it can resonate. However, the instructive interference of the coupled light with the third input port causes the light to propagate toward output ports. In this case the output power at the " $\mathrm{D}$ " and " $\mathrm{B}_{\text {out }}$ " are $65 \%$ and $84 \%$ respectively and can be considered logically as " 1 ". The EFD and spectrum response in this case are shown in Figs. 9 (a) and (b) correspondingly. 


\section{3. b) Time response analysis}

Nowadays technologies demand faster and more compact devices. Growing usage of internet at all over the world motivated the designer toward optical systems. As a result, all communications and calculations must be done optically and in a same frequency. The faster devices can improve the optical systems. Time response and device speed are among the criteria used for evaluation of the optical device behavior. The simulation results for seven cases of the proposed all-optical full-Subtractor are shown in Fig. 10

It can be seen from Fig. 10 that the full-Subtractor is very fast. The rise and fall time of Fig. 10 (a) are 2.8 and 0.17 ps respectively. This fast response allows doing more calculations in a specific time. The complete details of rising and falling times for all operation states are listed in Table 2.

The performance of the proposed full-Subtractor can be further investigated by calculating the contrast ratio (CR) which is the ratio of output powers of logic ' 1 ' and logic ' 0 ' as following [25]:

$C R=10 \log \left(P_{1} / P_{0}\right)$

where $P_{1}$ and $P_{0}$ in the above equation are the power values at the output port for logic ' 1 ' and logic ' 0 ', respectively. The average $\mathrm{CR}$ of the proposed half-Subtractor is greater than $10.35 \mathrm{~dB}$. The details of the CR for all eight states are brought in Table 2.

Table 2. Details of the characteristics of the proposed all-optical Full-Subtractor

\begin{tabular}{|c|c|c|c|c|c|c|c|c|}
\hline A & B & $B_{\text {in }}$ & $D \%$ & $\mathrm{~B}_{\text {out }} \%$ & $\mathrm{t}_{\mathrm{r}}(\mathrm{Ps})$ & $t_{f}(P s)$ & $\mathrm{CR}(\mathrm{db})$ & Worst Case \\
\hline 0 & 0 & 0 & 0 & 0 & - & - & - & \multirow{8}{*}{$10 \log _{10}\left(\frac{\operatorname{Min}(1)}{\operatorname{Max}(0)}\right)=$} \\
\hline 0 & 0 & 1 & $65 \equiv 1$ & $72 \equiv 1$ & 2.88 & 0.17 & - & \\
\hline 0 & 1 & 0 & $65 \equiv 1$ & $72 \equiv 1$ & 2.88 & 0.17 & - & \\
\hline 0 & 1 & 1 & $5 \equiv 0$ & $110 \equiv 1$ & 2.8 & 0.1 & 13.42 & \\
\hline 1 & 0 & 0 & $156 \equiv 1$ & $5 \equiv 0$ & 0.93 & 0.08 & 31.93 & \\
\hline 1 & 0 & 1 & $0.8 \equiv 0$ & $0.8 \equiv 0$ & 0.48 & 0.27 & - & \\
\hline 1 & 1 & 0 & $0.8 \equiv 0$ & $0.8 \equiv 0$ & 0.47 & 0.27 & - & \\
\hline 1 & 1 & 1 & $190 \equiv 1$ & $78 \equiv 1$ & 2.66 & 0.17 & - & \\
\hline
\end{tabular}

So far, no Full-Subtractor construction or design has been reported, so a comparison has been made between our proposed design and the reported Half-Subtractor or Full-Adders. The results are shown in Table 4. It can be seen that the results of our work are better or comparable to the other reported works

Table 3. Comparison between our proposed Full-Subtractor and some previous presented works. 


\begin{tabular}{|lllll|}
\hline Device Type & Rise time $(\mathrm{ps})$ & Fall Time $(\mathrm{ps})$ & $\mathrm{CR}(\mathrm{dB})$ & Ref. \\
\hline Half Sub. & 0.1 & - & 6.28 & {$[18]$} \\
\hline Half Sub. & 2 & 1 & 7.28 & {$[15]$} \\
\hline Half Sub. & 1 & 0.6 & - & {$[27]$} \\
\hline Full Adder & 0.6 & 0.36 & 4.77 & {$[12]$} \\
\hline Full Adder & 3 & - & 9.94 & {$[28]$} \\
\hline Full Sub. & 1.87 & 0.17 & 9.09 & This work \\
\hline
\end{tabular}

\section{Conclusion}

In this paper, as far as we known for the first time, we proposed a very simple device for realizing fullsubtraction behavior in a two-dimensional photonic crystal structure. To fulfill subtraction behavior, first, we used $33 \times 31$ silicon rods immersed in the air in a square lattice with lattice constant of $a=580 \mathrm{~nm}$. After that, we imposed some input and output waveguides, silicon defect rods, and one extra waveguide to the structure. Then we created a non-linear ring resonator to couple light. The role of defect rods are filtering and creating phase difference. The role of the extra output waveguide is to exhaust the light from the structure. The worst rise time and fall time of the device are $2.88 \mathrm{ps}$ and $0.27 \mathrm{ps}$ respectively and, so the proposed full-Subtractor is very fast. The worst CR of the proposed Subtractor is $10.35 \mathrm{~dB}$.

\section{Declarations}

"The authors declare no conflicts of interest."

\section{References}

1. E. Yablonovitch, "Inhibited spontaneous emission in solid-state physics and electronics," Phys. Rev. Lett., vol. 58, no. 20, pp. 2059, 1987.

2. Joannopoulos, J.D., Johnson, S.G., Winn, J.N., Meade, R.D.: Photonic Crystals: Molding the Flow of Light.

Princeton University Press, Princeton (2011).

3. Talebzadeh, R. and Soroosh, M., 2015. A dual cavity optical channel drop filter based on twodimensional photonic crystals. Journal of Optoelectronics and Advanced Materials, 17(11-12), pp.1593-1596.

4. Arjmand, M. and Talebzadeh, R., 2015. Optical filter based on photonic crystal resonant cavity. Optoelectronics and Advanced Materials-Rapid Communications, 9(1-2), pp.32-35.

5. Talebzadeh, R., Mehdizadeh, F. and Naseri, A., 2020. 4-channel tunable optical demultiplexer based on nonlinearity phenomenon in 2D resonant cavity photonic crystals. Frequenz, 74(1-2), pp.9-15. 
6. Goodarzi, K., Mir, A., 2015. Design and analysis of an all-optical De-multiplexer based on photonic Crystals. Infrared Physics and Technology, 68, pp. 193-196.

7. Talebzadeh, R., Soroosh, M., Kavian, Y.S. and Mehdizadeh, F., 2017. Eight-channel all-optical demultiplexer based on photonic crystal resonant cavities. Optik, 140, pp.331-337.

8. Talebzadeh, R., Soroosh, M. and Daghooghi, T., 2016. A 4-channel demultiplexer based on 2D photonic crystal using line defect resonant cavity. IETE journal of research, 62(6), pp.866-872.

9. Talebzadeh, R, Soroosh, M. and Mehdizadeh, F., 2016. Improved low channel spacing high quality factor four-channel demultiplexer based on photonic crystal ring resonators. Optica Applicata, 46(4).

10. Talebzadeh, R. and Soroosh, M., 2015. High quality complete coupling 4-channel demultiplexer based on photonic crystal ring resonators. Optoelectronics and Advanced Materials-Rapid Communications, 9(1-2).

11. Derakhshan, M., Naseri, A., Ghazizadeh, M. and Talebzadeh, R., 2018. Simulant designing of an ultracompact AND, OR logical gates based on two-dimensional photonic crystal waveguides. Photonic Network Communications, 36(3), pp.338-343.

12. A. M. Vali-Nasab, A. Mir, R. Talebzadeh, "Design and simulation of an all-optical full-adder based on photonic crystals." Optical and Quantum Electronics, Vol. 51(5), 2019, p.161.

13. Zhu, L., Mehdizadeh, F. and Talebzadeh, R., 2019. Application of photonic-crystal-based nonlinear ring resonators for realizing an all-optical comparator. Applied Optics, 58(30), pp.8316-8321.

14. K. Busch, "Photonic Crystals. Advances in design, Fabrication and Characterization” (Wiley, 6118).

15. R. Moradi, "All-optical half subtractor using photonic crystal based nonlinear ring resonators." Optical and Quantum Electronics, Vol. 51(4), 2019, p.119.

16. A. Askarian, G. Akbarizadeh, and M. Fartash, "All-optical half-subtractor based on photonic crystals. Applied optics," Vol. 58(22), 2019, pp. 5931-5935.

17. Namdari, N. and Talebzadeh, R., "Simple and compact optical half-subtractor based on photonic crystal resonant cavities in silicon rods," Applied Optics, Vol. 59(1), 2020, pp.165-170.

18. Parandin, F., Malmir, M.R. and Naseri, M., "All-optical half-subtractor with low-time delay based on two-dimensional photonic crystals. Superlattices and Microstructures," Vol. 109, 2017, pp.437-441.

19. Boyd RW. Nonlinear optics. Academic press; 2019 Aug 15.

20. Williams DJ, Prasad P. Introduction to nonlinear optical effects in molecules and polymers. New York: Wiley; 1990.

21. Johnson, S., Joannopoulos, J.: Block-iterative frequency-domain methods for Maxwell's equations in a planewave basis. Opt. Express 8, 173 (2001).

22. W. Bogaerts, P.D. Heyn, T.V. Vaerenbergh, K.S. DeVos, K. Selvaraja, T. Claes, P. Dumon, P. Bienstman, D. Van Thourhout, R. Baets, Silicon microring resonators, Laser Photon. Rev. 6 (2012) 47-73.

23. Green, M.A., Keevers, M.J.: Optical properties of intrinsic silicon at 300 K. Prog. Photovolt. 3, 189-192 (1995). 
24. Taflove, A.: Computational Electrodynamics: The Finite Difference Time domain Method. Artech House, Norwood (1995).

25. Tang, C., Dou, X., Lin, Y., Yin, H., Wu, B., Zhao, Q.: Design of all-optical logic gates avoiding external phase shifters in a two-dimensional photonic crystal based on multi-mode interference for BPSK signals. Opt. Commun. 316, 49-55 (2014).

26. V. François, S. Najafi, T. Ohtsuki, N. Peyghambarian, S. Honkanen, S. Lalrenière1 and M. Andrews, "Progress towards realisation of rare-earth-doped glass integrated-optics lasers", Proc. SPIE 2213, Nanofabrication Technologies and Device Integration, (28 July 1994);

27. A. Askarian, G. Akbarizadeh and M. Fartash, "A novel proposal for all optical half-subtractor based on photonic crystals". Optical and Quantum Electronics, Vol. 51(8), 2019, p. 264.

28. M. Moradi, M. Danaie, A.A. Orouji, "Design and analysis of an optical full-adder based on nonlinear photonic crystal ring resonators", Optik, 2018.

\section{Figures}

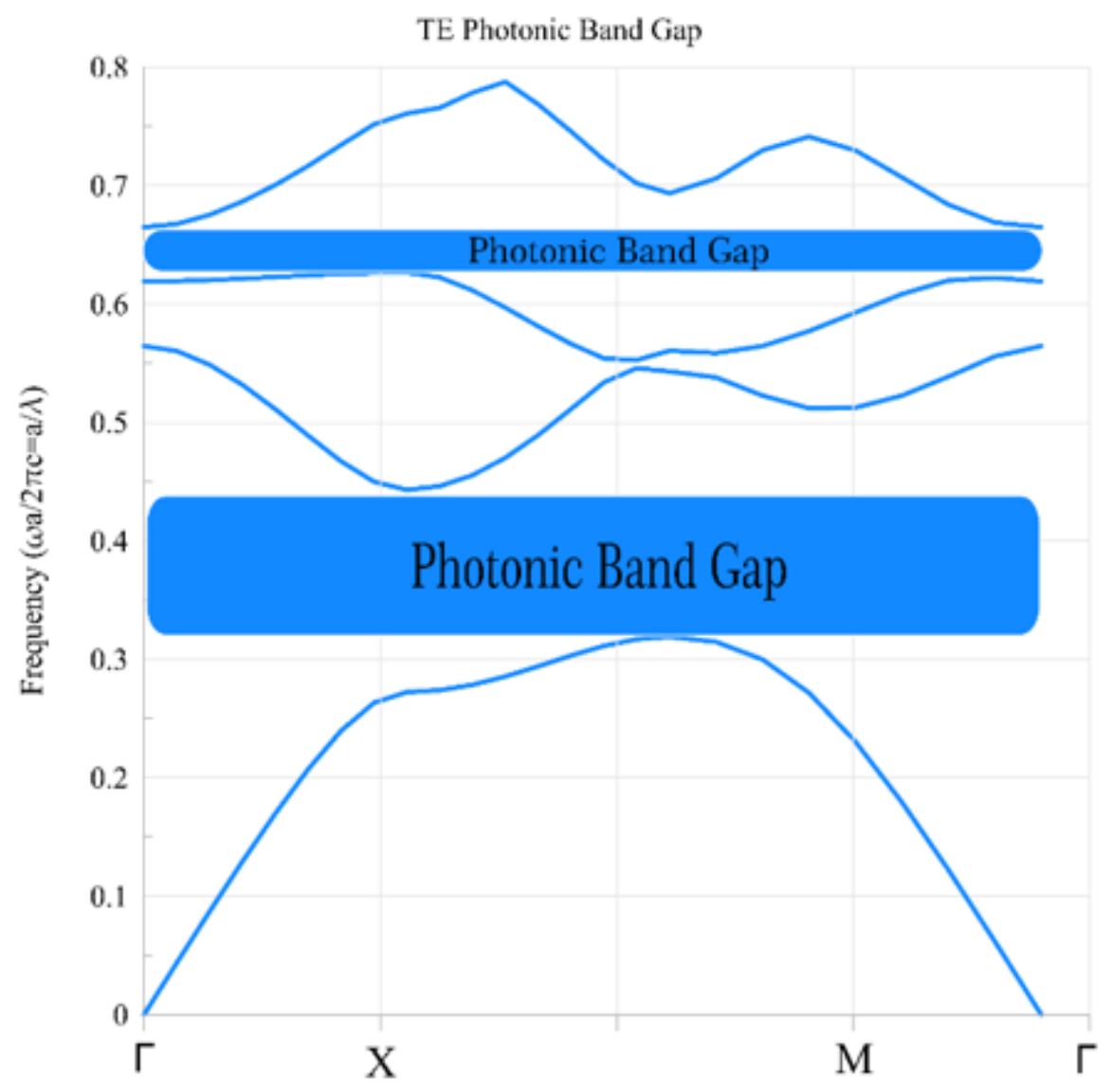

Figure 1

The band structure and PBGs of the basic proposed photonic crystals arrangement. 


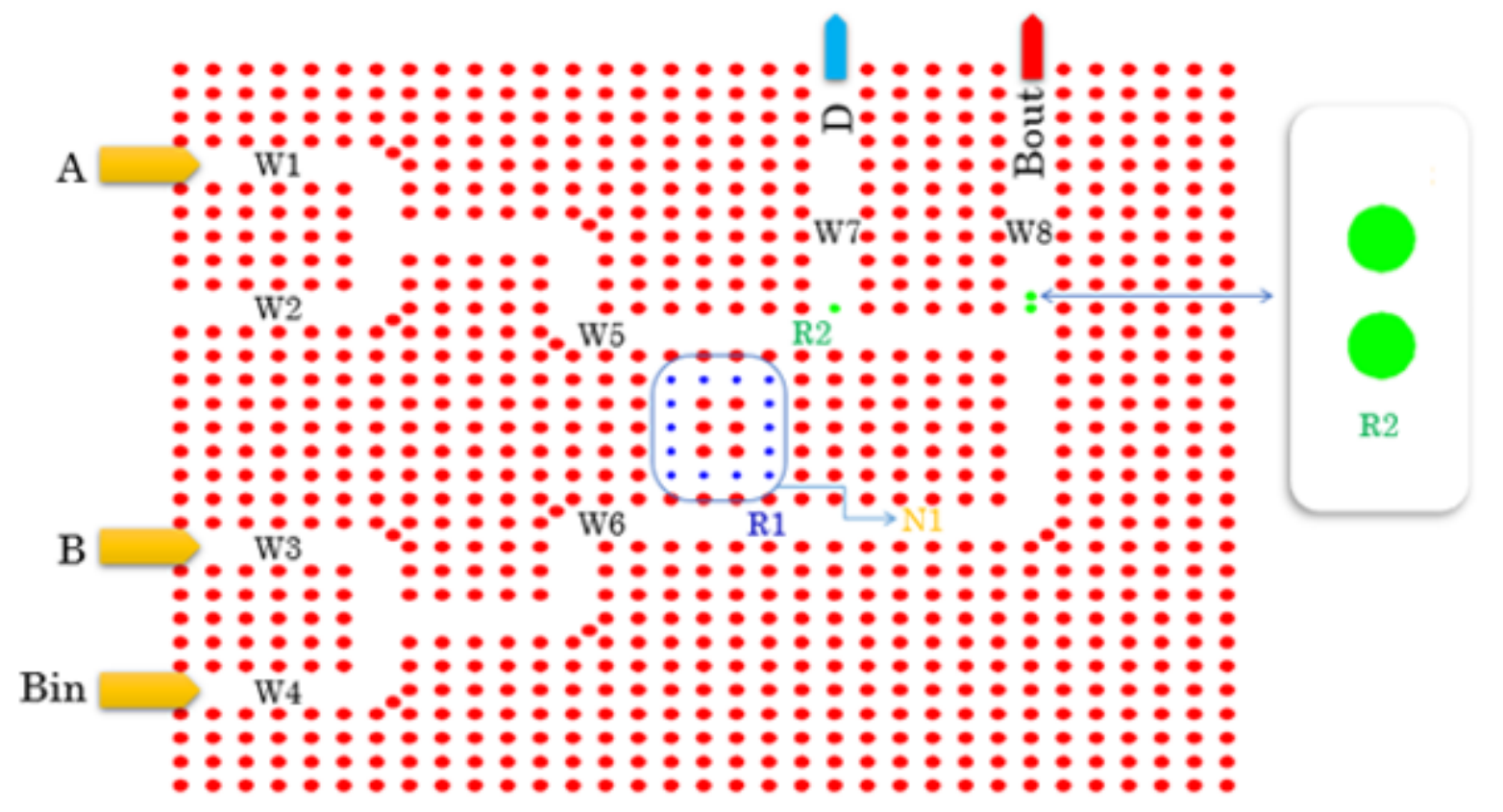

Figure 2

The schematic diagram of the proposed Full-Subtractor.

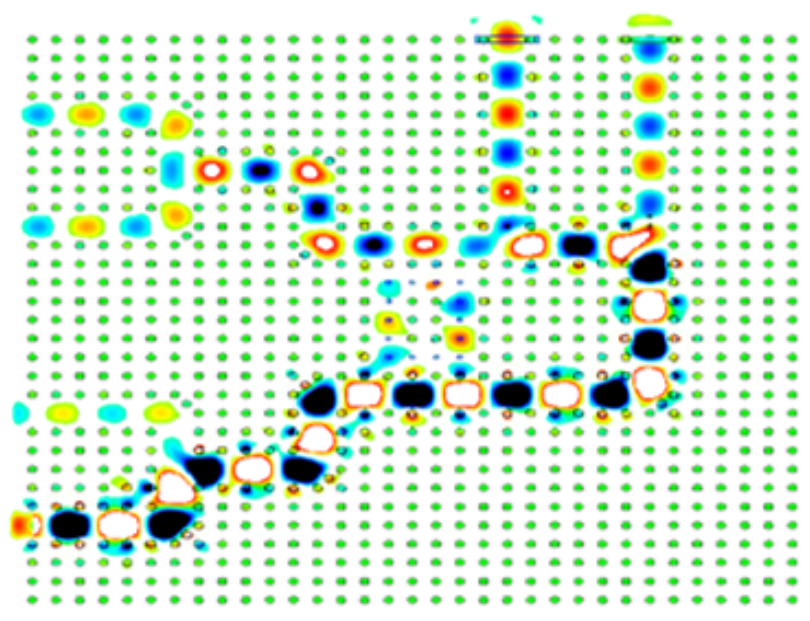

(a)
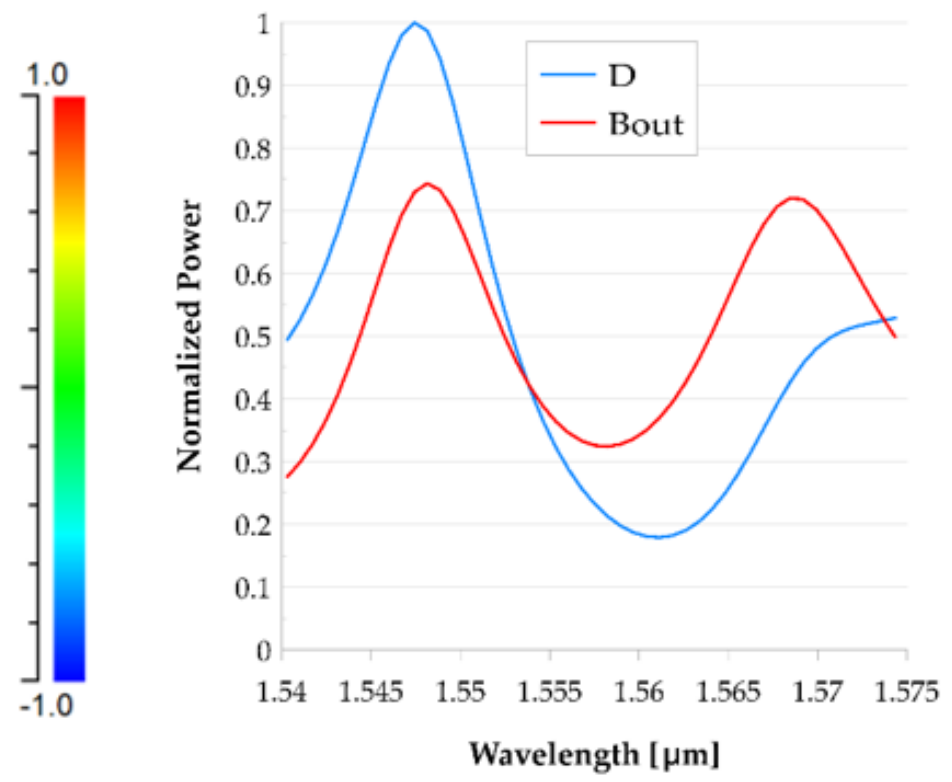

(b)

Figure 3

a) The EFD, and b) Output spectrum of the proposed full-Subtractor related to the case "A" ="B"= "0" and "Bin" = "1". 


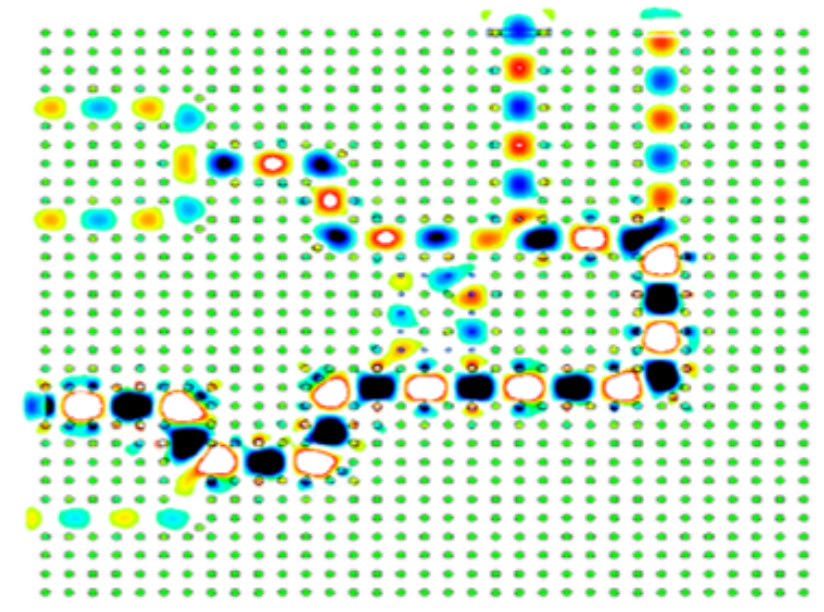

(a)
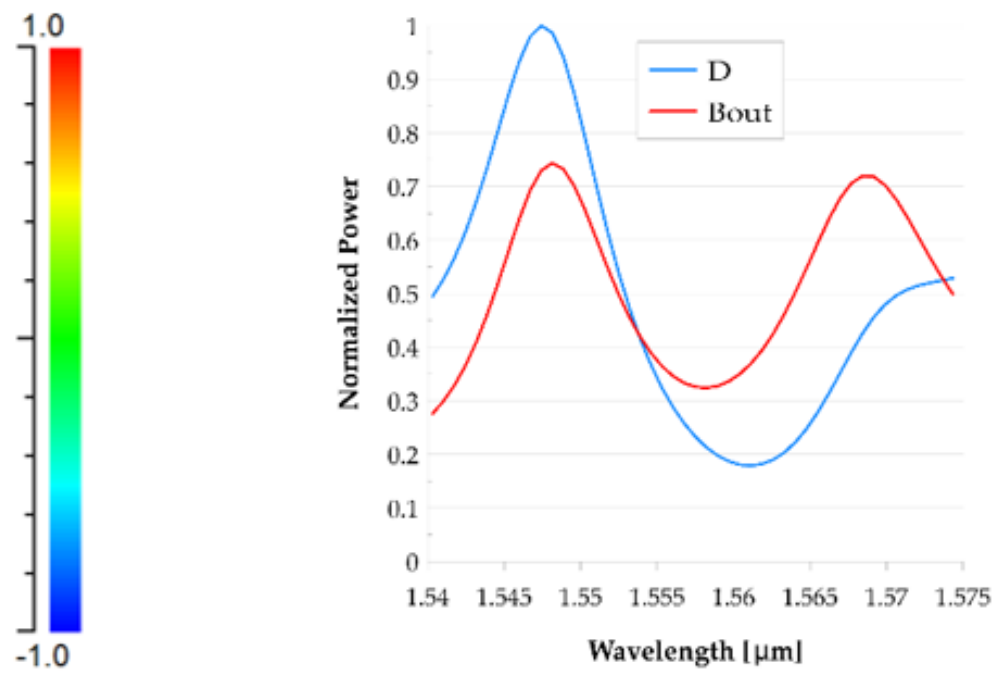

(b)

Figure 4

a) The EFD, and b) Output spectrum of the proposed full-Subtractor related to the case "A" ="Bin"= "0" and "B" = "1".

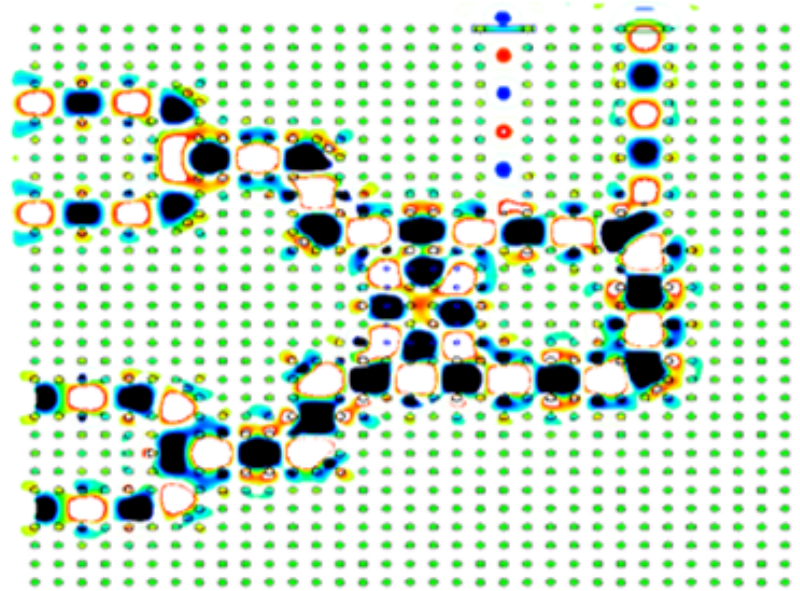

(a)
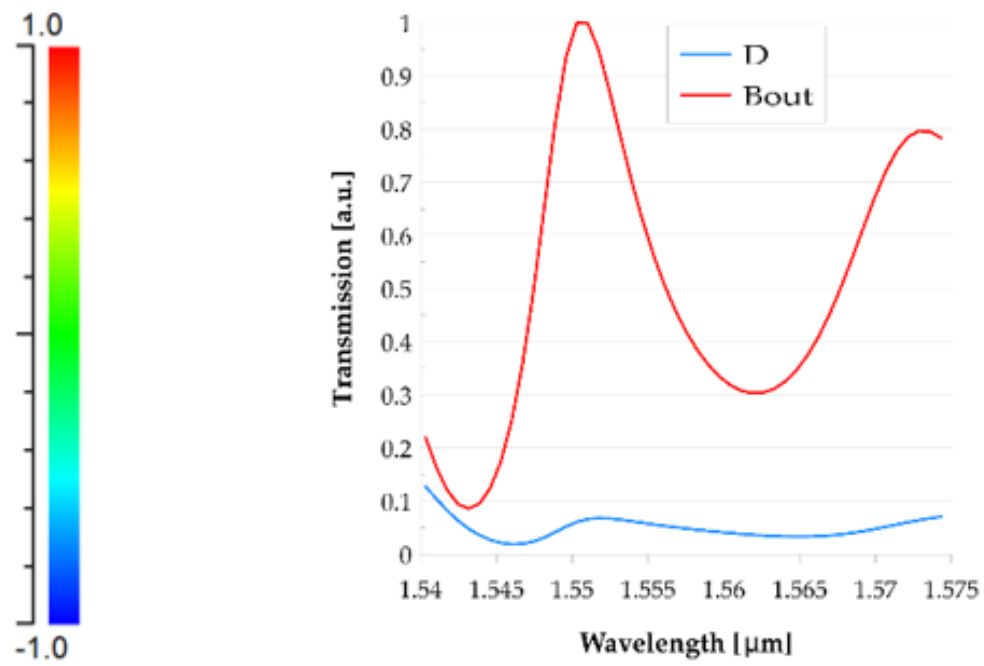

(b)

Figure 5

(a) The EFD and (b) Output spectrum of the proposed full-Subtractor related to the case "A" ="0" and "B"="Bin" = "1". 


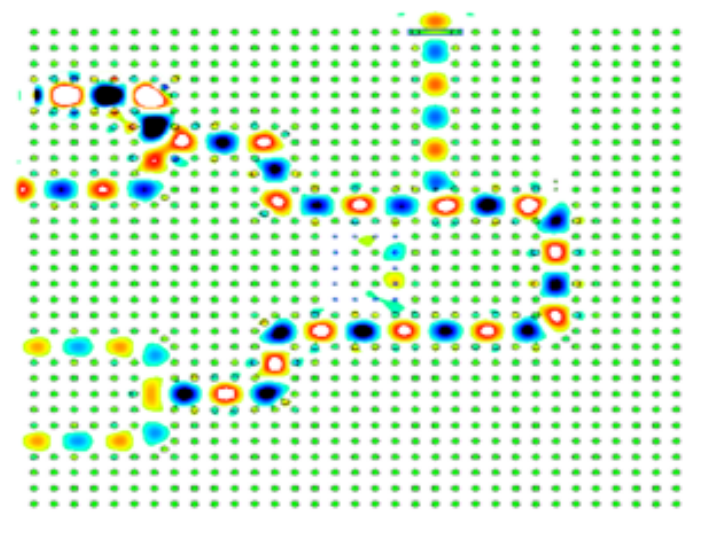

(a)
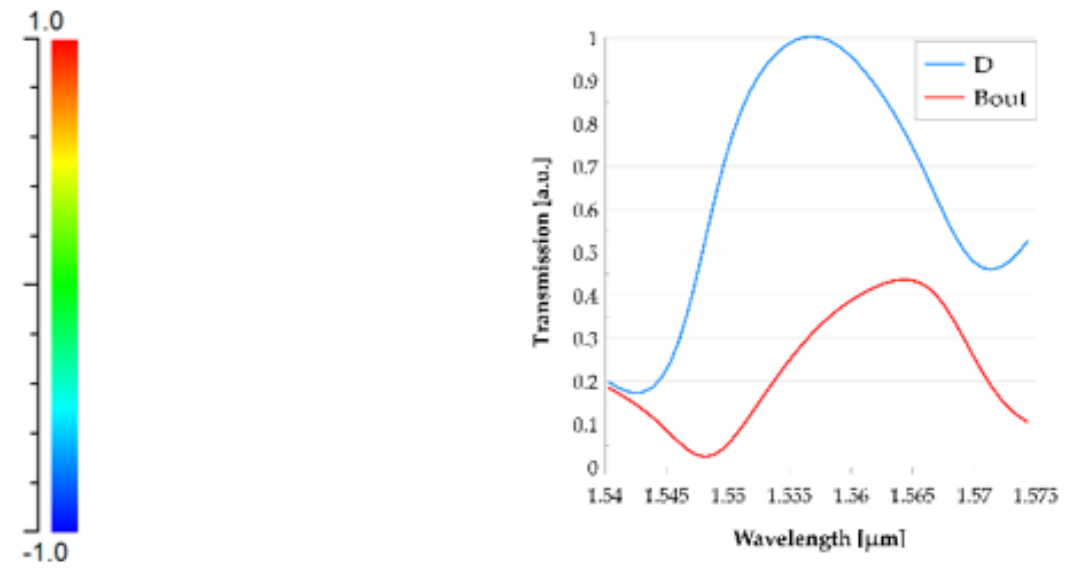

(b)

\section{Figure 6}

(a) The EFD and (b) Output spectrum of the proposed full-Subtractor related to the case "A" =" 1 " and "B"="Bin" = "0".

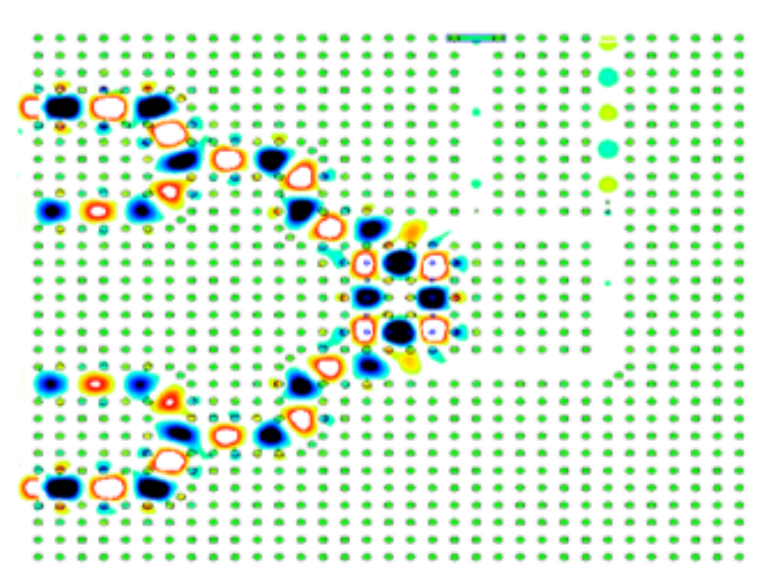

(a)

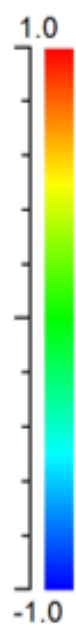

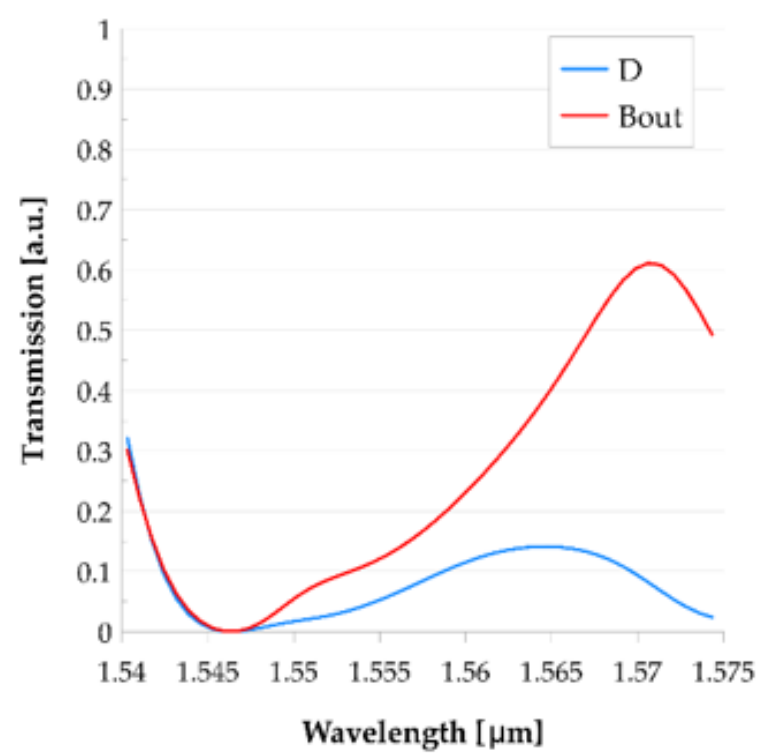

(b)

\section{Figure 7}

(a) The EFD and b) Output spectrum of the proposed full-Subtractor related to the case "A" ="1", "B"="0", and "Bin" = "1". 


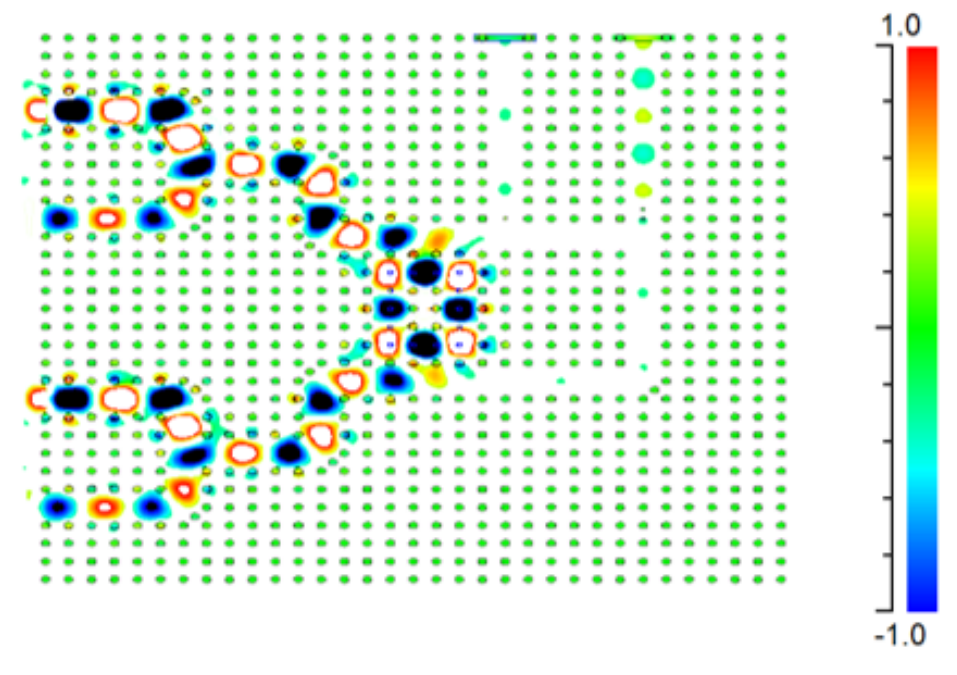

(a)

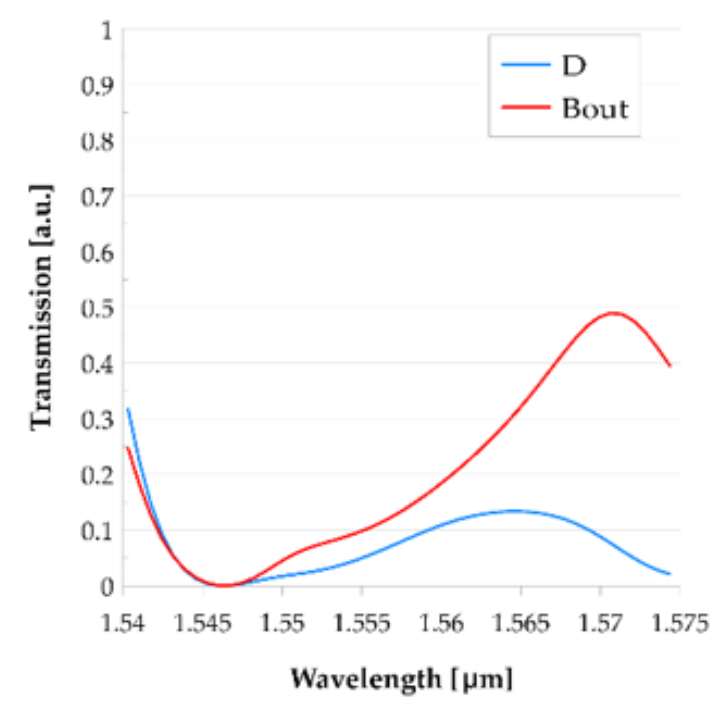

(b)

Figure 8

(a) The EFD and (b) Output spectrum of the proposed full-Subtractor related to the case "A" = "B"= "1", and "Bin" = "0".

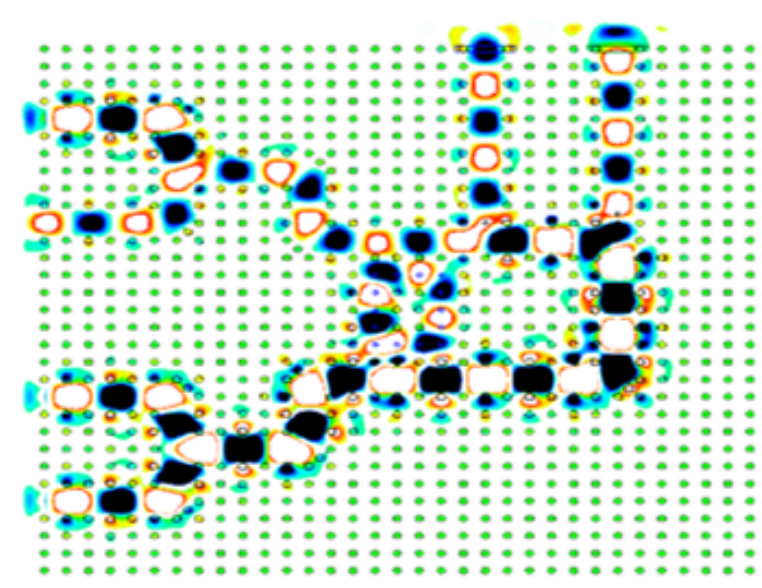

(a)
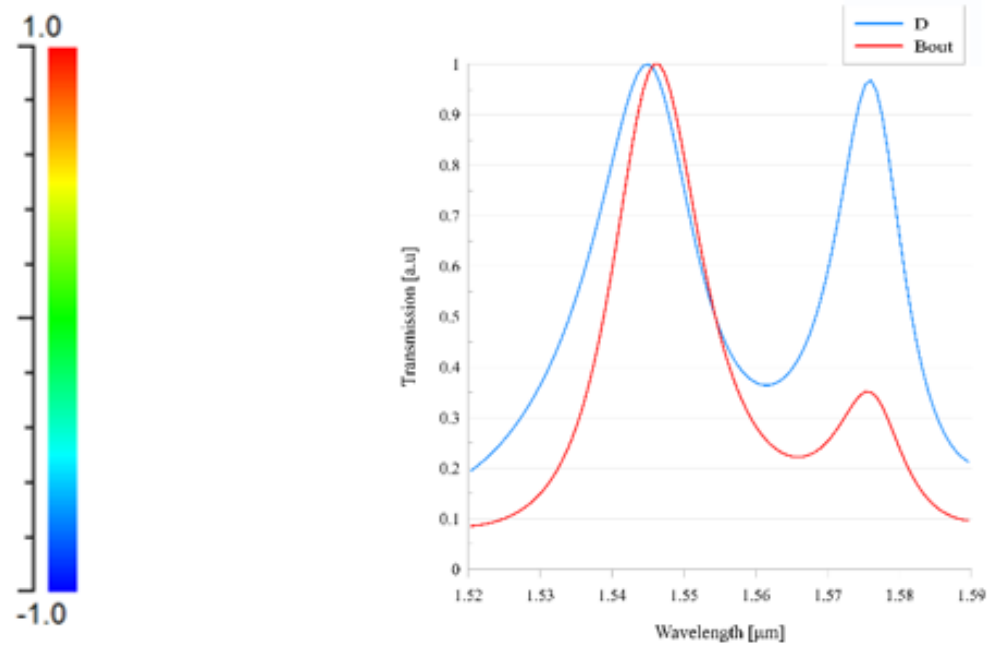

(b)

Figure 9

(a) The EFD and (b) Output spectrum of the proposed full-Subtractor related to the case "A" = "B"= "Bin" = "1". 

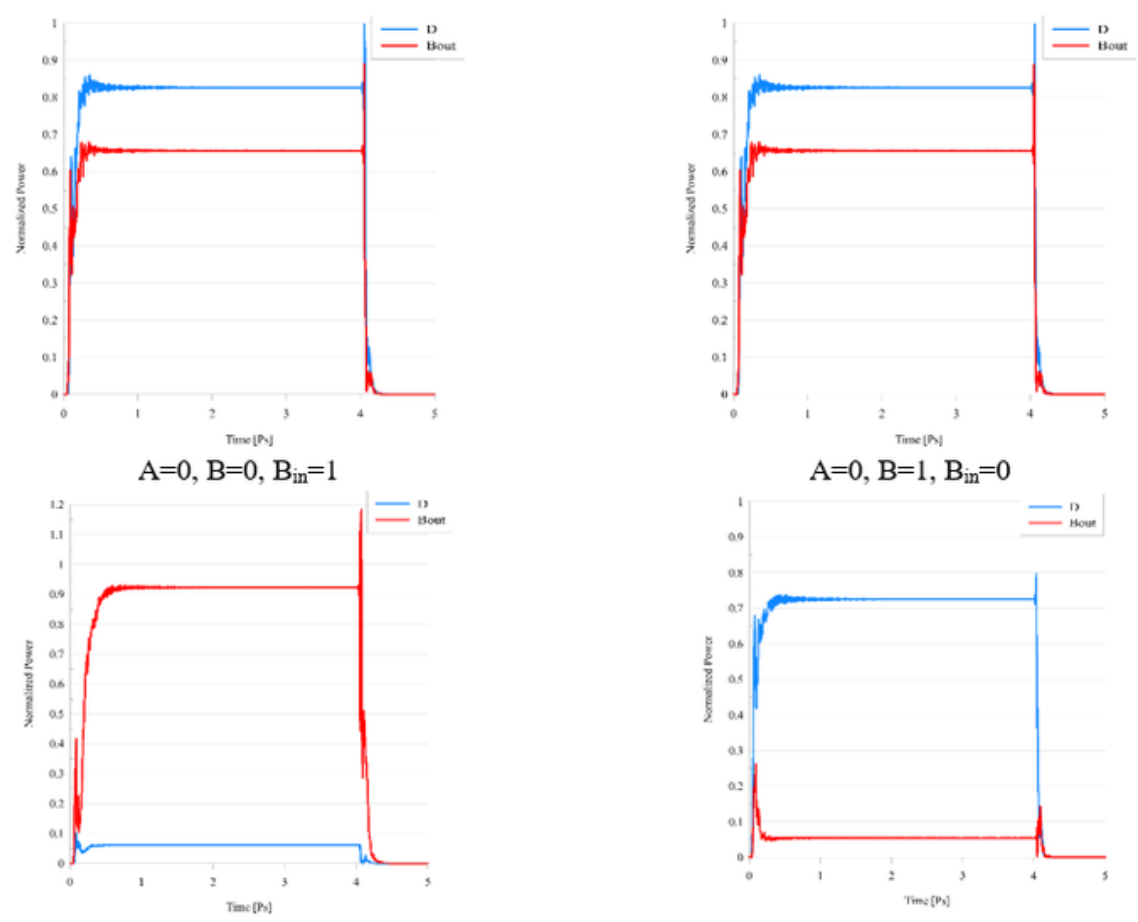

$\mathrm{A}=0, \mathrm{~B}=1, \mathrm{~B}_{\mathrm{in}}=0$

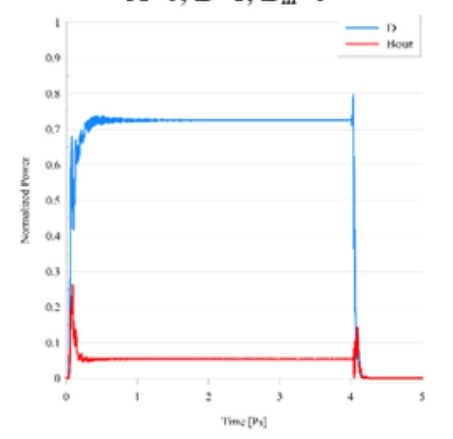

$\mathrm{A}=0, \mathrm{~B}=1, \mathrm{~B}_{\mathrm{in}}=1$

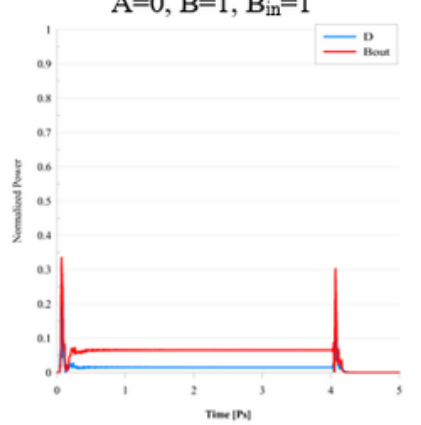

$\mathrm{A}=1, \mathrm{~B}=0, \mathrm{~B}_{\text {in }}=1$
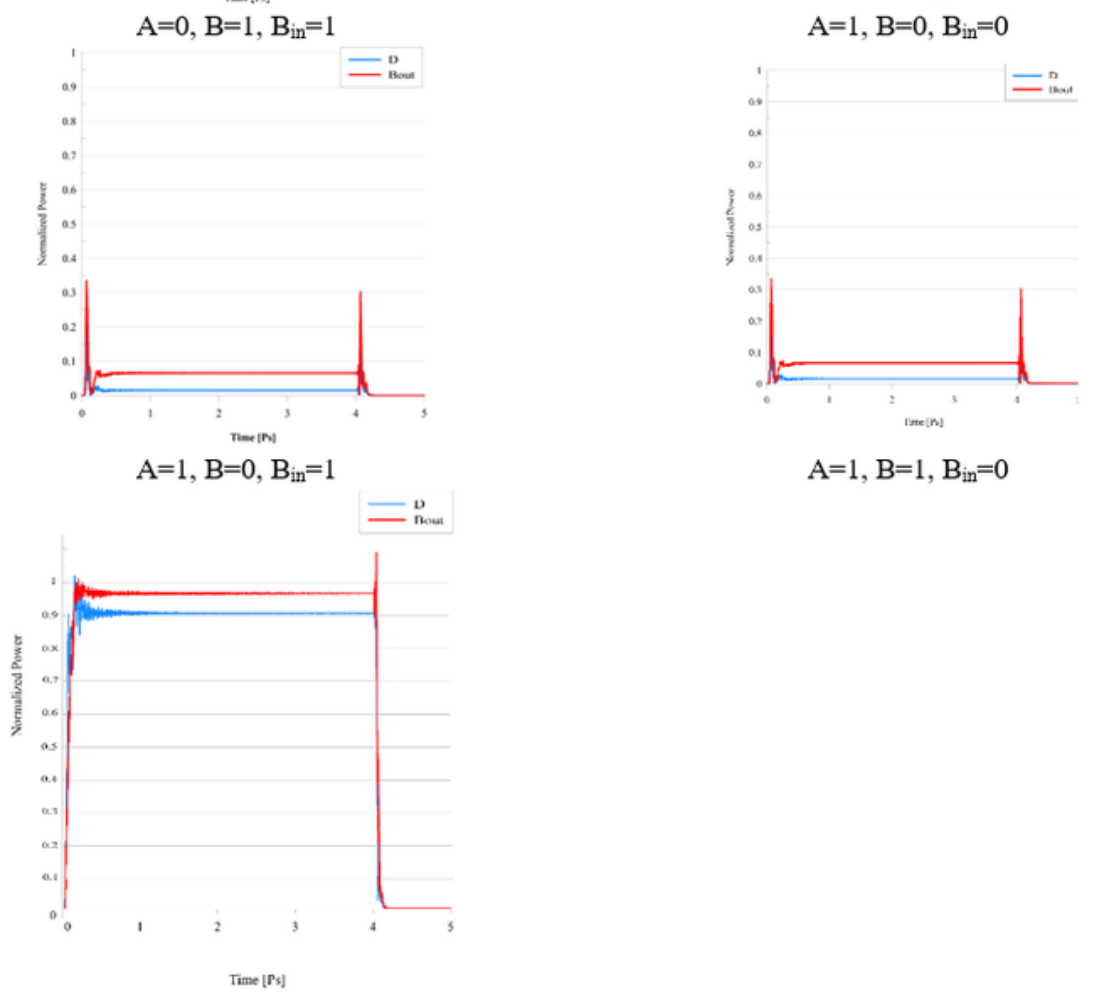

$\mathrm{A}=1, \mathrm{~B}=1, \mathrm{~B}_{\text {in }}=0$

$\mathrm{A}=1, \mathrm{~B}=1, \mathrm{~B}_{\mathrm{in}}=1$

\section{Figure 10}

Time response of the two outputs of the proposed all-optical full-Subtractor for all seven states. 